Recepción: 08 / 04 / 2018

Aceptación: 10 / 06 / 2018

Publicación: 15 / 09 / 2018
Ciencia de la computación Artículo de investigación

\title{
Servicios y aplicaciones de voz sobre ip utilizando el estándar H.323
}

\author{
Voice over ip services and applications using the H.323 standard
}

Serviços de voz sobre IP e aplicativos usando o padrão H.323

\author{
Javier Guaña-Moya ${ }^{\mathrm{I}}$ \\ eguana953@puce.edu.ec \\ Viena Muirragui-Irrazábal II \\ vmuirraguii1@unemi.edu.ec
}

\section{Correspondencia: eguana953@puce.edu.ec}

${ }^{I}$ Doctor en Ciencias Informáticas, Docente de la Pontificia Universidad Católica del Ecuador, Quito, Ecuador.

II Master en Gerencia de Tecnologías, Docente de la Universidad Estatal de Milagro, Guayas, Ecuador. 


\title{
Resumen
}

La telefonía IP es una alternativa sobre la telefonía tradicional por la reducción de costos en sus llamadas, por lo que los bits transmitidos entre extensiones utilizando dispositivos multiVoIP (MVP) es de 96 bits y la trasmisión de dos extensiones (terminal A al B) utilizando dos MVP es de 292 bits por cada llamada, dando así un $67.1 \%$ de ahorro en la trasmisión de datos en cada llamada bajo IP. La comunicación telefónica con tecnologías IP, utiliza protocolos orientados y no orientados a comunicación, por cuanto con TPC/IP se trasmite 40 paquetes, dando un $10.5 \%$ de la comunicación entre dos extensiones; mientras que UDP (no orientado a comunicación) ocupa 357 paquetes, esto equivale a un $89.5 \%$ de la comunicación, aseverando así que la mayoría de paquetes utilizados son con UDP, por lo que la comunicación en llamadas bajo IP, no tienen un gran porcentaje de seguridad en los datos trasmitidos.

Palabras clave: redes; voz sobre IP; estándares de red; VoIP, H.323.

\begin{abstract}
IP telephony is an alternative over traditional telephony due to the reduction of costs in its calls, so the bits transmitted between extensions using multiVoIP devices (MVP) is 96 bits and the transmission of two extensions (terminal A to B) using Two MVP is 292 bits per call, thus giving a $67.1 \%$ saving in data transmission in each call under IP. The telephone communication with IP technologies uses oriented and non-communication oriented protocols, since with TPC / IP 40 packets are transmitted, giving $10.5 \%$ of the communication between two extensions; while UDP (non-communication oriented) occupies 357 packets, this is equivalent to $89.5 \%$ of the communication, thus asserting that most of the packets used are UDP, so communication in low IP calls does not have a large percentage of security in the transmitted data.
\end{abstract}

Key words: networks; voice over IP; network standards; VoIP, H.323.

\section{Resumo}

A telefonia IP é uma alternativa à telefonia tradicional devido à redução de custos em suas chamadas, portanto os bits transmitidos entre ramais usando dispositivos multiVoIP (MVP) são de 96 bits e a transmissão de duas ramais (terminal A para B) usando Dois MVP são 292 bits por chamada, proporcionando uma economia de $67,1 \%$ na transmissão de dados em cada chamada 
sob IP. A comunicação telefônica com tecnologias IP utiliza protocolos orientados e não orientados à comunicação, uma vez que com o TPC / IP são transmitidos 40 pacotes, dando $10,5 \%$ da comunicação entre duas extensões; enquanto UDP (non-communication oriented) ocupa 357 pacotes, isto equivale a 89,5\% da comunicação, afirmando assim que a maioria dos pacotes usados são UDP, então a comunicação em chamadas IP baixas não tem uma grande porcentagem de segurança nos dados transmitidos.

Palavras chave: redes; voz sobre IP; padrões de rede; VoIP, H.323.

\section{Introducción}

La evolución de las comunicaciones, así como también el impacto de la tecnología en todo el mundo, ha permitido disminuir la brecha digital en los sectores más vulnerables, logrando así cambios sustanciales en la sociedad, alcanzando innovación constante en las aplicaciones tecnológicas y el paso de la comunicación telefónica tradicional a una comunicación más económica y al alcance del usuario en general.

Según, las redes de comunicaciones están constituidas por varios ordenadores y sistemas operativos heterogéneos, los cuales están conectados a la red de internet, mismos que permiten un mejor desarrollo en la comunicación, acortar distancias y generar una comunicación permanente y en tiempo real.

Estas redes de comunicaciones han permitido generar nuevos procesos de innovación en la telefonía, logrando así una evolución constante de la telefonía alámbrica e inalámbrica, minimizando costos en la comunicación y mejorando su calidad en las llamadas a través de líneas dedicadas. Estos nuevos avances han generado las comunicaciones denominadas Voz sobre IP o también llamada VoIP, la cual es una tecnología que permite la transmisión de fragmentos auditivos, digitalizados a través de la red de Internet, por lo que se puede describir que la transmisión de Voz sobre la infraestructura de red ha generado grandes expectativas, por el ahorro de recursos que ésta tecnología representa, es por ello que al utilizar esta red de VoIP permitirá generar nuevas aplicaciones de voz, datos y video bajo IP, generando un ahorro sustancial de recursos físicos y monetarios, y al mismo tiempo facilitando el uso de la 
infraestructura de red, logrando así un mejor rendimiento de los proceso en la red IP y la utilización de todos sus recursos y aplicaciones.

Los estándares utilizados en VoIP fueron determinados por la UIT-T para establecer una base común para la comunicación abierta en todos los fabricantes, por lo cual VoIP trabaja con estándares como el H.323, H.320, etc., mismos que establecen un mecanismo de comunicación e implantación de la voz sobre redes IP.

Es importante describir que redes IP, han permitido el ingreso de nuevos protocolos y estándares de comunicación, por ello se puede decir que las soluciones de primera instancia estaban basadas en tecnologías propias o propietarias; ya que dichas tecnologías no permitían comunicarse entre sistemas de diferentes fabricantes y se denominaban arquitectura cerrada, por ello VoIP permite integrar diferentes plataformas telefónicas que existen en las instituciones públicas y privadas, logrando así una comunicación abierta y escalable.

Por todo lo dicho, es importante recalcar que la Voz sobre IP tiene cuatro elementos importantes que son cliente, servidor, Gateway y equipo final.

Cliente: se presenta como una aplicación a través de software corriendo en una PC controlada mediante una interface gráfica (GUI); así como también como un cliente "virtual" que reside en el Gateway y tienen las siguientes características:

Transmisión: Establece la comunicación, codifica, empaqueta y transmite al medio de propagación.

Recepción: Realiza el proceso inverso. Decodifica y reproduce la señal de voz a través de los audífonos o parlantes.

Servidor: Los servidores realizan varios procesos como operaciones complejas de bases de datos, manejo de dominios, servidores proxy, etc., dichas operaciones se realizan en tiempo real y de forma local. 
Gateways: Son dispositivos que realizan un puente de comunicación entre los usuarios, los cuales permiten unir la red VoIP con la red telefónica analógica PSTN (Public Switched Telephone Network).

Teléfonos: Son dispositivos finales denominados teléfonos Normal y teléfono IP.

En cuanto a los estándares de VoIP, H.323 permite comunicaciones multimedia y garantiza una calidad de servicio (QoS), combinada con adaptadores, Gateway y otros productos de infraestructuras, permiten una conectividad universal dentro y fuera del ámbito de una misma institución y está compuesto por: Extremo, Gatekeeper (GK), Gateway (GW), Unidad de Control Multipunto (MCU), Terminal y Procesador Multipunto (MP).

Un punto importante en las comunicaciones es la calidad de servicio (QoS), lo que está definido como la capacidad de la red para administrar el tráfico que se genera en la trasmisión y recepción de datos, lo que permite satisfacer las necesidades del usuario en la transmitir voz sobre IP, lo cual permite mejorar el rendimiento de la red y garantizar la comunicación.

\section{Métodos}

El presente artículo fue realizado mediante una investigación acerca de la tecnología VoIP, redes de área local, aplicaciones de voz sobre IP, estándares de comunicación, etc. Dicha información proporcionó datos necesarios sobre las tendencias actuales de la tecnología VoIP y sus estándares, así como también del modo de configuración de un Work Station en forma de router, para generar el control del envío y recepción de datos.

Para los procesos de transmisión y comunicación entre el cliente y el servidor, así como también una comunicación con calidad de servicio y seguridad en la red de datos, se configuro dos equipos Multitech entre los cuales se utilizó el MVP 400 como server y el equipo MVP 200 como cliente.

Los equipos Multitech fueron configurados con el Estándar H323 para el uso de todas las aplicaciones que los dispositivos permiten, ya que por defecto vienen estructurados con estándares propietarios, los cuales tienen limitación en las aplicaciones IP. 
Se configuró un Work Station para que tenga bondades de un router con el fin de permitir la transmisión de información en la red. Dicha estación está configurada con dos tarjetas Fastethernet, las cuales permiten el paso de dos segmentos de red, una pasarela del servidor y la otra del cliente en dicha estación, también se configuro el Ethereal que permite el monitoreo del filtrado de paquetes.

Los equipos MVP fueron configurados para realizar multillamadas y utilizar el servicio de fax utilizando la red VOIP, también fueron configurados para permitir la trasmisión de voz bajo la red IP con teléfonos normales, estos permitirán utilizar todos los servicios convergentes de la red y el abaratamiento de costos por las aplicaciones de cada equipo.

\section{Resultados}

Como resultados de la investigación se describe a continuación las diferentes configuraciones y aplicaciones de los equipos MultiVOIP (Multitech):

\section{Configuración del MultiVOIP MVP400 y MVP200 (Host)}

Para configurar los equipos MultiVOIP se realizó una conexión de red local, lo que permitió que los equipos sean configurados mediante consola (TELNET), es importante recalcar que los dispositivos también pueden ser configurados de forma gráfico. Para generar la comunicación entre el MVP y el equipo, se asignó direcciones IP con su respectiva Máscara a los dispositivos Multitech, así como también a la estación base para la comunicación, logrando así que todos los equipos estén en la misma red, lo que permitirá el acceso a la configuración de los dispositivos antes mencionados.

Es importante configurar el Estándar de comunicación, por ello se configuro el estándar H.323, el cual define a los Sistemas de Comunicaciones como transmisión multimedia basados en paquetes, por lo que dicho estándar permite tener una arquitectura distribuida, la cual genera aplicaciones de forma multimedia, así como también la comunicación de VoIP.

La configuración del MVP 200 como host consiste en instalar el Oem, Coders, H323 Stack, Factory defaults y Firmware. Es importante describir que en el momento de añadir extensiones a 
este equipo hay que tener en cuenta la configuración como Host y se activa el Propietary Phone Book, el que permitirá ingresar el número para la comunicación.

\section{Monitoreo y filtrado de paquetes utilizando ethereal}

En la Fig. 1, se muestra una topología en estrella, la cual permitirá realizar el monitoreo y filtrado de paquetes, la red local estará en un solo dominio de Broadcast en todos los puertos y estarán conectados a los equipos MultiVoIP y al Work Station que contiene el Sistema Operativo Fedora Core 4.0. EL equipo servidor tiene dos interfaces (eth0 y eth1) para realizar la función de un router y así poder recibir y enviar paquetes de diferentes redes (LAN, MAN o WAN).

La Work Station deberá estar configurado como un router y con un software para el monitoreo y filtrado de paquetes como el Ethereal que al utilizarlo se obtendrá el tráfico existente en la red cuando se esté realizando una llamada telefónica desde los equipos MultiVoIP.

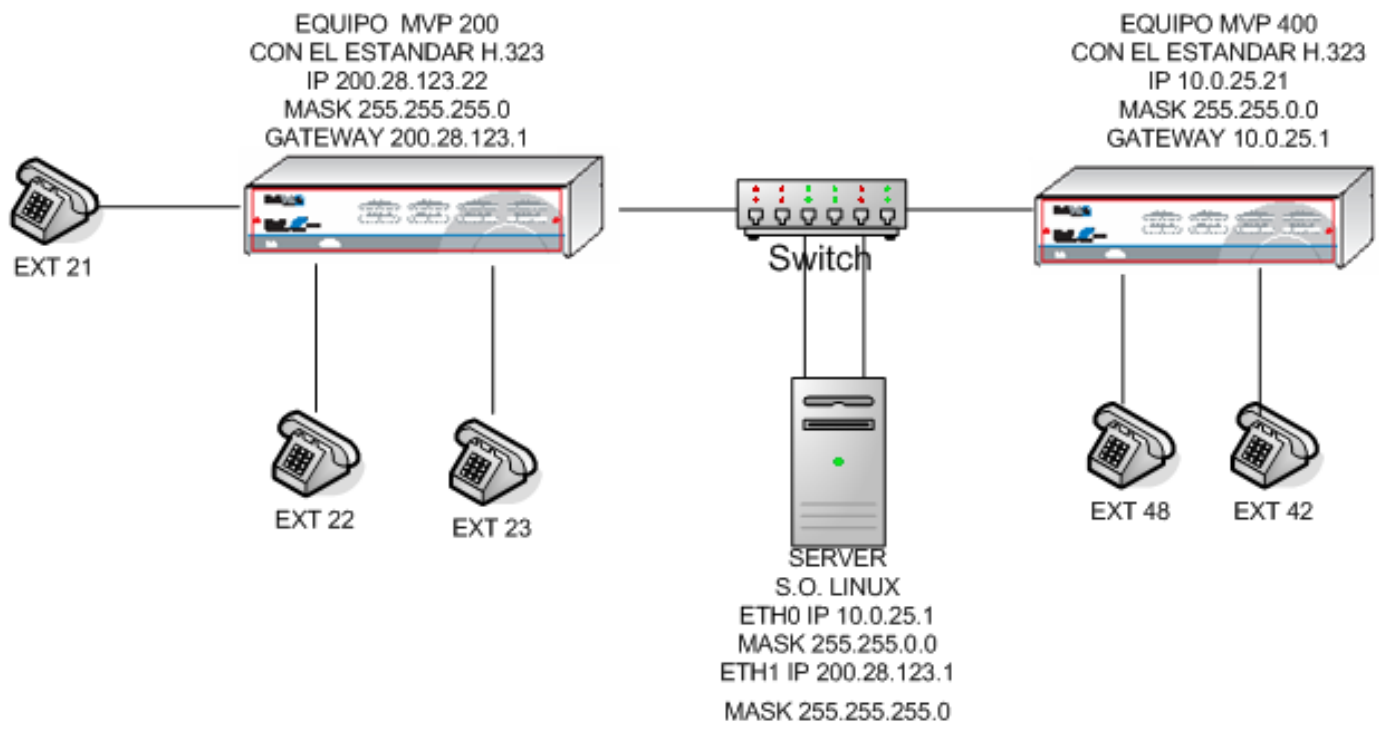

Fig. 1 Diseño de la red para monitoreo y filtrar los paquetes 
Una vez realizado la instalación del Sistema Operativo Fedora Core 4.0, y el Sistema Operativo Windows para realizar la configuración de los equipos MultiVoIP se tiene que utilizar los siguientes requerimientos:

\section{Server}

•eth0 Link encap: Ethernet HW addr 00:20:78: 15:6 B: D6

inet addr:10.0.25.1 Bcast:10.0.255.255 Mask:255.255.0.0

•eth1 Link encap: Ethernet HW addr 00:90:27:1B:09: ED

inet addr:200.28.123.1 Bcast:200.28.123.255 Mask:255.255.255.0

\section{Equipos Multitech}

•MVP 200: IP: 200.28.123.22 Mask: 255.255.255.0, Gateway 200.28.123.1

•MVP 400: IP: 10.0.25.21 Mask: 255.255.0.0, Gateway 10.0.25.1

\section{Llamas, Multillamadas y envío de fax con equipos MVP - VoIP}

Para las llamadas, comunicación y configuración con equipos VoIP se utilizó, 4 teléfonos análogos, 2 Fax y dos tarjetas de red para el monitoreo. Toda esta implementación se ha realizado en una topología de red en estrella; también se ha instalado el Sistema Operativo Fedora Core 4.0 en un Work Station, para obtener el Monitoreo y Filtrado de Paquetes TCP/IP para visualizar la información que se está transmitiendo en la comunicación VoIP.

En la Fig. 2 se muestra una topología de red en la cual existen teléfonos análogos conectados a los equipos (MVP 200 y 400), estos a su vez conectados mediante un switch o hub a un Work Station para realizar las pruebas necesarias en la comunicación con el estándar H.323.

Para realizar las pruebas en llamadas y comprobar su efectividad en los equipos, se debe obtener una comunicación clara a través de los equipos MVP, para ello se describe los siguientes pasos:

\section{Usuario A llamando Usuario B}

-Usuario A marca el número de extensión del usuario B requerido.

-La extensión llamada timbra. 
-El abonado llamado contesta.

-Se establece la conexión y posteriormente para finalizar la llamada cerrar los teléfonos.

Remarcación de usuario (A-B).

-Usuario A marcar el número de extensión del usuario B requerido.

-La extensión llamada timbra.

-El abonado llamado no contesta.

-El usuario A cierra el teléfono.

- Para volver a llamar al usuario B se debe presionar el radial.

-La extensión llamada timbra.

-Finalmente se establece la conexión.

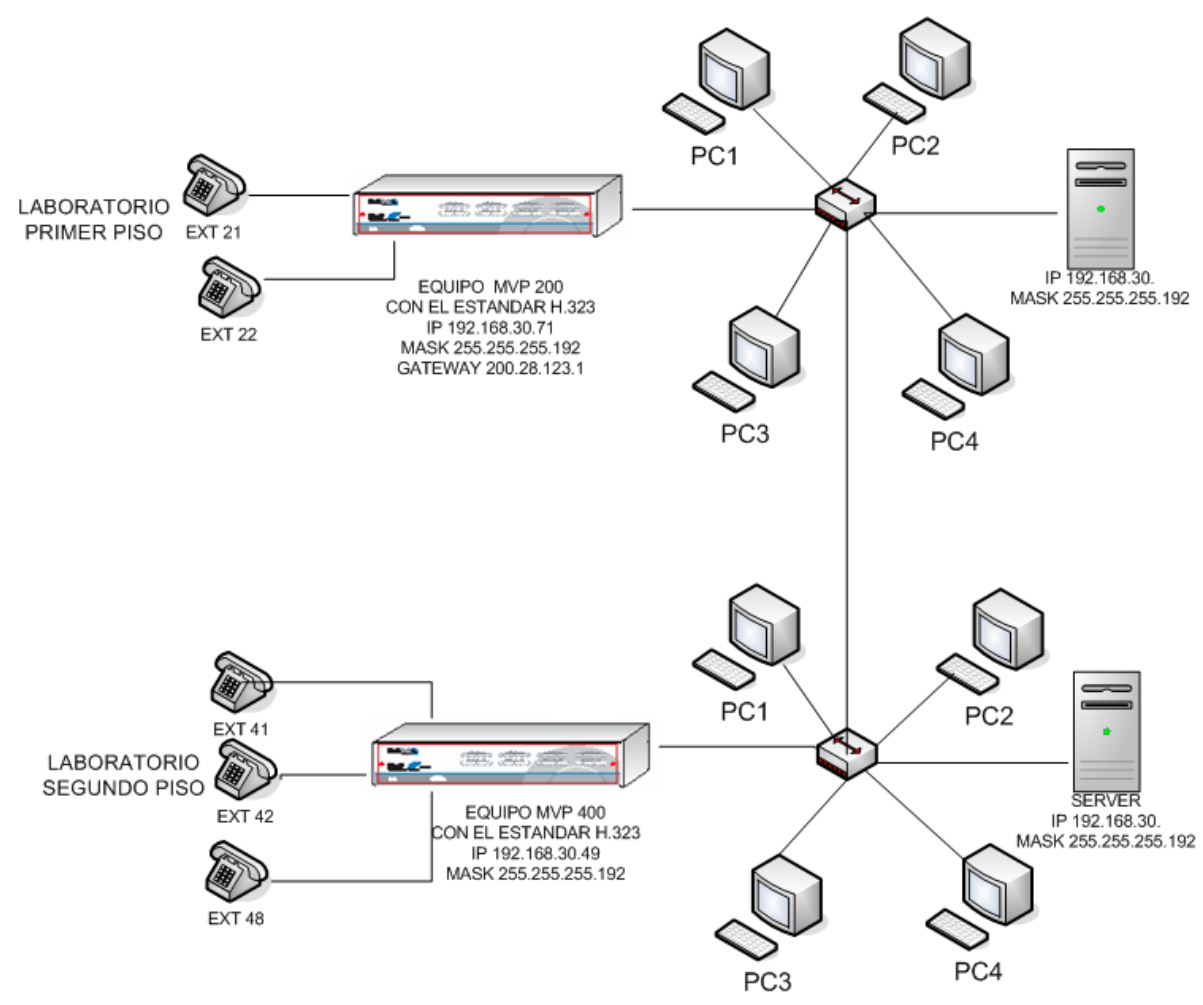

Fig. 2 Topología de red con equipos MVP 
Para realizar las multillamadas se debe configurar los equipos en una de las dos formas existentes:

Si están en la misma red se configurará uno como HOST otro como CLIENTE.

Mientras que, si están en dos redes diferentes, se deberá establecer a los dos equipos como HOST individuales para que cada uno trabaje con su propio directorio telefónico y permita enrutar correctamente los paquetes, para así tener una excelente comunicación.

Para las multillamadas se debe conectar los teléfonos en todos los puertos FXS de los MVP como se muestra en la figura 2, esto permitirá realizar llamadas y enviar Fax simultáneamente, así como también comprobar su efectividad.

En la Fig. 3 se muestra las conexiones de una topología en estrella entre la zona 1 y la zona 2 con una serie de teléfonos utilizando todos los puertos FXS de los equipos MVP.

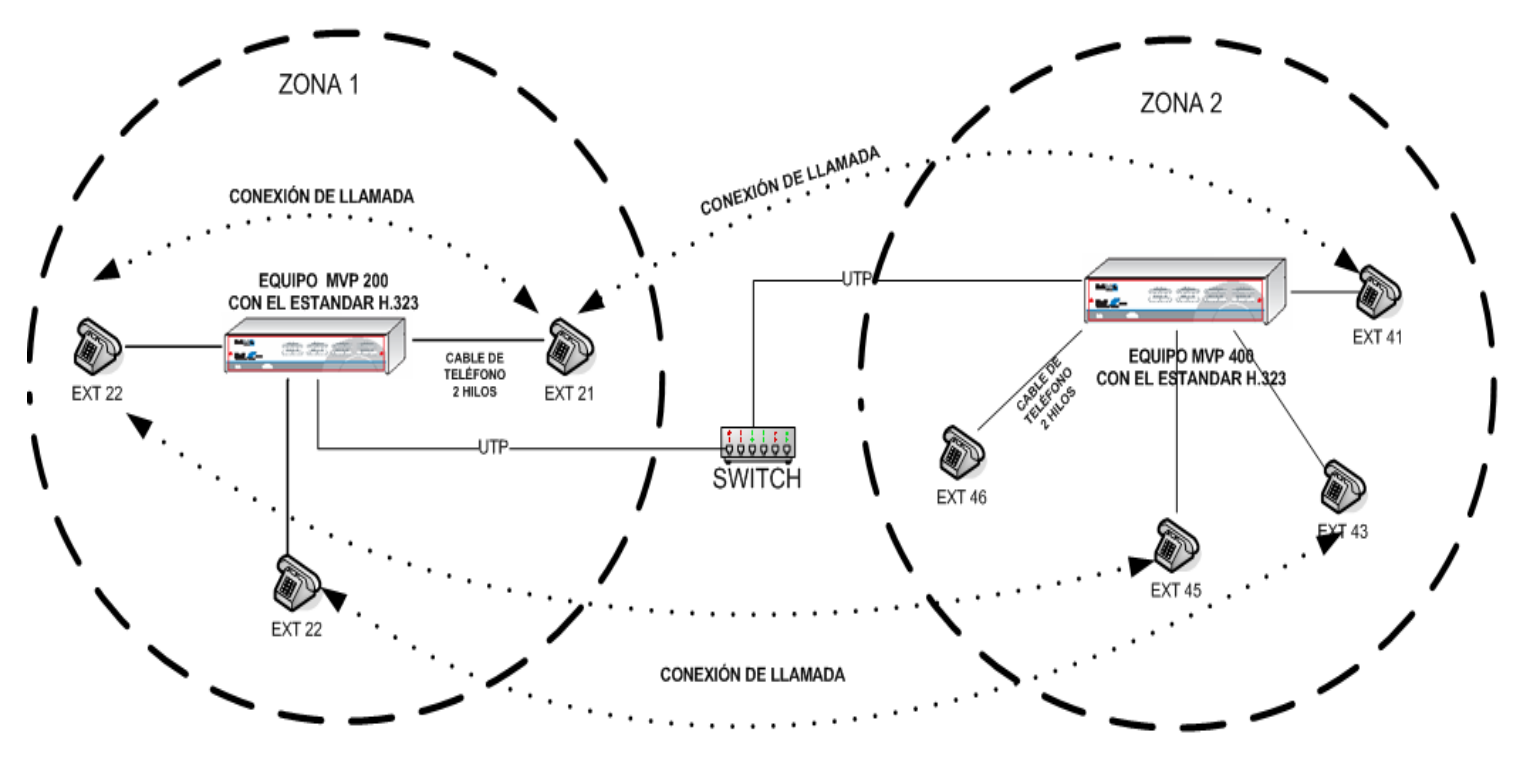

Fig. 3. Multillamadas con los equipos MVP

Es importante resaltar que la fidelidad de la comunicación en las llamadas puede limitarse por características propias de los equipos y de la red LAN. 


\section{Para la Configuración de fax se debe ingresar los parámetros que se describen a continuación:}

\section{Seleccionar el Canal 1}

Ingresar el ancho de banda máxima que soporta el equipo

Duración máxima que tendrá el envío de fax

Conectar los equipos de Fax en los puertos FXS, en el equipo MVP 400 en el canal 1 que está configurado como (extensión 41), y en el MVP 200 en el canal 1 (extensión 21)

Realizar la llamada telefónica normal de la extensión 40 a la extensión 21.

Después de tener conexión pedir tono de Fax y cerrar los teléfonos

Dejar que los equipos de Fax procesen su información para que posteriormente los equipos MVP hagan su trabajo con el protocolo T.38.

Finalmente esperar que el documento del Fax llegue a su destino y recibir el acuse de envío en el Fax conectado en el puerto 1 del MVP como Extensión 40.

En cuanto a los envíos y recepción de Fax, en la Fig. 4, se visualiza el envío de Fax de usuario A - B, los cuales están transmitiendo información utilizando un solo equipo (MVP200), así como también en la trasmisión de los dos MVP (200 y 400), los cuales se describen a continuación:

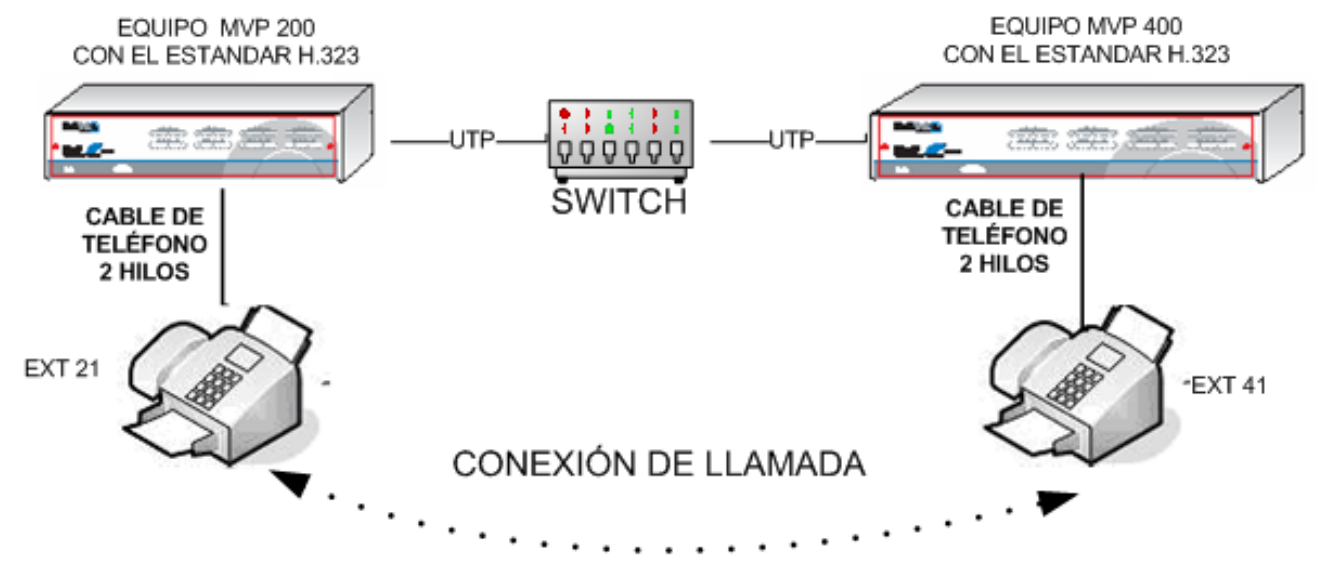

Fig. 4. Envío de fax con dispositivos MVP 


\section{Conclusiones}

Poseer tecnologías y estándares propietarios restringe el campo de las comunicaciones, ya que corre el peligro de que los dispositivos se queden aislados, esto depende directamente de los clientes y proveedores, por lo que los equipos con el estándar H.323, permiten interoperabilidad entre los distintos fabricantes, de hecho, compañías como Cisco y otros operadores mundiales han agregado a sus diferentes productos de comercialización.

La telefonía IP es una alternativa sobre la telefonía tradicional por la reducción de costos que implica en las llamadas, así como también la calidad de servicios (QoS) en sus comunicaciones y el cifrado de la información obtenida, por lo cual la comunicación bajo IP disminuye un 50\% en costos de llamadas, así como también la infraestructura de las redes telefónicas convencionales.

Los bits que se transmiten en la comunicación entre teléfonos utilizando un solo MVP es de 96 bits; mientras que la trasmisión de un terminal A al B, utilizando el equipo MVP 400 y el MVP 200 es de 292 bits por cada llamada, dando así un $67.1 \%$ de ahorro en la trasmisión de datos en cada llamada bajo IP.

En cuanto a los protocolos de comunicación en cada llamada, el protocolo TPC/IP trasmite 40 paquetes, dando así un $10.5 \%$ de la comunicación; mientras que UDP ocupa 357 paquetes, esto equivale a un $89.5 \%$ de la comunicación, esto confirma que la mayoría de paquetes utilizados son protocolos no orientado a comunicación (UDP), por lo que la comunicación en llamadas bajo IP, no tienen un gran porcentaje de seguridad en los datos trasmitidos.

\section{Referencias Bibliográficas}

Vera, A. S. (2016). Diseño e implementación de un prototipo nodo multimedia para servicios de voz sobre IP, navegación por internet. Repositorio Institucional Universidad de Cuenca.

Dordoigne, J. (2015). Redes informáticas-Nociones fundamentales (5ª edición): (Protocolos, Arquitecturas, Redes inalámbricas, Virtualización, Seguridad, IP v6...). Ediciones ENI.

Martelo, R. J. (2015). Metodología para Seleccionar Aplicaciones de la Tecnología de Comunicaciones Voz sobre la IP (VoIP) para Pequeñas y Medianas Empresas (Pymes). Información tecnológica., 121-128. 
Jiménez, C. \&. (2017). Solución para llamadas de voz sobre IP mediante la utilización de Software Libre. Organismo público en Costa Rica (Doctoral dissertation).

Martín, M. J. (2015). Fortalecimiento de las redes académicas de voz sobre IP latinoamericanas para una integración sostenible y sustentable. Segunda Conferencia de Directores de TIC de Instituciones de Educación Superior.

Joskowicz, J. (2013). Voz, video y telefonía sobre IP. Instituto de Ingeniería Eléctrica, Facultad de Ingeniería. Montevideo Uruguay

Ferreira, J. C. (2016). Evaluación del rendimiento de una red LAN sobre power line communications para la transmisión de VOIP. Innovación e Investigación en Ingeniería, 83-95.

Ligña, C., \& Humberto, L. (2014). Diseño de una red de voz sobre IP para la empresa Egar SA (Bachelor's thesis, Quito: Universidad de las Américas, 2014.). 